\title{
La vigencia de la ética normativa
}

\section{Validity of normative ethics}

Ana Patricia Melchor Organista Universidad Autónoma Metropolitana ann.pmo29@gmail.com

\section{Resumen}

En el presente texto se busca cumplir dos objetivos: en la primera parte se argumenta a favor de retomar los trabajos de ética normativa, así como de la necesidad de vincularlos activamente con las investigaciones en metaética y ética aplicada. En segundo lugar, se traza una precaución fundamental que debe ser tomada en cuenta al momento de hacer tanto ética normativa como ética aplicada, ilustrada con el caso de la ablación femenina en poblaciones africanas.

Palabras clave: Ética Normativa, Intuiciones Morales, Ética Aplicada, Ablación Femenina. 


\begin{abstract}
The present text aims towards two main goals: in a first moment, it argues in favour of going back to the normative ethics researches and the necessity to actively link them with metaethics and applied ethics researches. The second part outlines a fundamental precaution that needs to be taken into account while making normative or applied ethics, illustrated with the case of female circumcision in African countries.
\end{abstract}

Key words: Normative Ethics, Moral Intuitions, Applied Ethics, Female Circumcision.

\title{
Introducción
}

Existen pocos temas más escabrosos dentro de la filosofía que los que se relacionan con la moralidad y es que no existe unanimidad sobre qué es lo moral o qué implicaciones tiene. En nuestro día a día vemos cómo se habla de «lo bueno», «lo correcto», de «los valores»y, si se tiene algún entrenamiento en la tarea filosófica, ante cada enunciación que apele a estos conceptos presuponiendo su significado, no podrá evitar sentir el impulso de querer golpear su cabeza contra la pared.

El presente texto no pretende responder a las preguntas acerca del significado último de esos conceptos, no podría. Su meta es meramente proponer una dinámica distinta entre los niveles de análisis en ética que, con algo de 
suerte, nos permitirá arrojar algo de luz a las discusiones más apremiantes en sus tres niveles. Por último, traza una precaución necesaria al momento de analizar y postular teorías o sistemas morales, que sirve a su vez para mostrar la complejidad de la tarea a la que nos enfrentamos todos los que deseemos continuar explorando las discusiones éticas.

Para la primera sección, se presentan los tres niveles de análisis de la ética: la metaética, la ética normativa y la ética aplicada, sus objetos de estudio y la relación que existe entre ellos. Después se profundiza en la ética normativa, en la forma en que se ha dado respuesta a sus preguntas, las críticas principales que se le han hecho y se argumentará que, a pesar de todos los problemas que presenta, es necesario retomarla y vincularla con los otros dos niveles de la ética.

En la segunda sección se analiza la forma en que conceptos reconocidos como propiamente «éticos» han sido empleados por distintas organizaciones y gobiernos para intervenir en países africanos y cómo una situación que, a primera vista, va claramente en contra del bienestar de un grupo «vulnerable», no es simple. Es necesario analizar a fondo las circunstancias del caso, ampliar el alcance de nuestra reflexión y evitar que la normatividad a la que aspiramos esté sesgada y sea capaz de enfrentarse a la complejidad de casos como el de la ablación femenina. 


\section{Los niveles de análisis en ética y el papel de la Ética Normativa}

Dentro de la ética, existen tres niveles principales de análisis: la metaética, la ética normativa y la ética aplicada. La distinción clásica señala que la ética normativa y la metaética son dos niveles distintos de estudio de la moral: la ética normativa ofrece respuestas a cómo se debería actuar, establece principios normativos o máximas para la acción y la metaética analiza los elementos de la ética normativa, específicamente, lo que se quiere decir cuando se emplean ciertos conceptos problemáticos, como «lo bueno» o «lo correcto». La ética aplicada se dedica a buscar respuestas para preguntas puntuales sobre discusiones de casos en las que se emplean términos y principios morales.

De esta forma, la ética normativa establece guías para la acción; la metaética analiza tales guías, de dónde vienen y qué significan, y la ética aplicada busca emplear elementos normativos para problemas específicos que enfrentamos como sociedad. Las preguntas de la ética normativa son de primer orden: preguntas sobre cómo deberíamos actuar; las de la metaética son de segundo orden: preguntas sobre cómo establecemos que debemos actuar de cierta forma, $\mathrm{y}$ las preguntas de la ética aplicada son de primer orden y específicas a casos particulares, qué tendríamos qué hacer frente una situación $x$, que necesita una resolución pronta y posiblemente sui generis. 
Idealmente, la relación entre los tres niveles podría ser lineal, es decir, las conclusiones en el nivel metaético, acerca del significado del lenguaje moral y de nuestra psicología moral, tendrían que servir de base para establecer una teoría normativa exitosa y la teoría normativa establecida tendría que darnos las herramientas necesarias para resolver los problemas que se plantea la ética aplicada. Como puede preverse, ese no es el caso e incluso hay problemas de comunicación entre los tres niveles de análisis, quien hace ética aplicada pocas ocasiones se concentra en el significado preciso de la terminología que emplea y quien hace metaética no suele involucrarse en los debates que ocupan a la ética aplicada.

Existe además una tendencia a dejar de lado las aspiraciones de la ética normativa, el proyecto de encontrar una norma o serie de normas que le den a cada agente pautas para actuar moralmente y le permitan estar justificado en su acción parece no llevar a resultados exitosos que puedan enfrentarse a las principales críticas hechas a las teorías normativas. Su búsqueda de universalidad, de ser aplicables sin que las circunstancias de la situación y del sujeto intervengan en el análisis, resulta más de lo que las teorías normativas, en sus versiones originales o reformuladas, pueden lograr.

Para comprender la aparente falla de la ética normativa es necesario comprender a fondo cuáles son las metas a las que aspira y una forma de acercarnos a la 
dimensión propiamente normativa de la ética es analizar cuáles son las preguntas que formula y desde dónde las formula. Christine Korsgaard presenta la pregunta normativa y sostiene que cada sistema moral debe responder a ella, algunos de forma más exitosa que otros. Se trata de preguntar qué es lo correcto por hacer ante una situación específica y, sobre todo, de justificar esa respuesta: «Una teoría moral normativa debe permitirnos actuar a la luz de un pleno conocimiento de lo que es la moralidad y de por qué somos sensibles a su influencia, y al mismo tiempo permitirnos creer que nuestras acciones están justificadas y tienen sentido» (Korsgaard, 2000, 31).

La pregunta normativa se formula desde la primera persona y surge cuando un agente se encuentra ante un requerimiento de la moral que va en contra de lo que dicho agente estaría inclinado a realizar; la respuesta a la pregunta normativa tendría que explicar qué es lo que la moral requiere y por qué dicho curso de acción está justificado ${ }^{1}$.

La clasificación tradicional de las teorías normativas divide las teorías en tres: las deontológicas, las

${ }^{1}$ La clasificación de las propuestas morales de Korsgaard difiere de la tradicional, ella divide a las teorías morales en las voluntaristas, las que siguen un realismo sustantivo, las que apelan a un "asentimiento reflexivo» y las que apelan a la autonomía. Cada una responde a la pregunta normativa de forma más o menos exitosa. Aquí nos concentramos en la clasificación clásica para abordar las críticas que tradicionalmente se le han hecho a la ética normativa. 
consecuencialistas y la ética de la virtud ${ }^{2}$. La presentación de los tres sistemas que se hace a continuación se concentra en sus postulados principales desde sus defensores clásicos, si bien cada teoría ha cambiado en la actualidad, conforme sus defensores buscan responder a las objeciones que se les plantean.

El consecuencialista sostiene que una acción moralmente correcta se define por sus consecuencias, si las consecuencias de una acción son deseables, entonces es correcta. El utilitarismo es una forma de consecuencialismo, y conforme a él la evaluación de una acción se concentra en el placer o felicidad que resultan de ella: si el resultado de la acción es el mayor número de felicidad para el mayor número de personas, entonces ése es el curso de acción que debe tomarse.

El sistema deontológico se concentra en el deber que tiene el agente para actuar de una forma específica: una acción será moralmente correcta si pasa la prueba de racionalidad práctica y lo que la impulse sea el deber, que surge de la razón. La formulación más conocida y a la que más se recurre es que uno debe actuar de tal forma que la máxima que guía la acción que se evalúa pueda convertirse en principio de acción para todos los demás, si las razones

2 Si bien una crítica importante que suele hacérsele es que no se trata propiamente de un sistema normativo, sino más bien de consejos prudenciales para la acción y no está formulada como una norma universal; la incluimos en tanto proporciona pautas para la conducta. 
que se tienen para actuar son universalizables. Otra formulación importante es que debemos tratar a los demás siempre como fines en sí mismos y nunca sólo como medios. Si seguimos el Imperativo Categórico podremos actuar correctamente.

La ética de la virtud sostiene que lo que hace que un acto sea moralmente correcto es que sea realizado por un agente virtuoso. El enfoque de esta teoría se centra en las características del agente que actúa, señalando que la práctica de la virtud es la que hace que un hombre sea virtuoso. La virtud es concebida como el justo medio entre el defecto y el exceso. Aristóteles parte de una concepción de los hombres como seres que buscan la felicidad y la identifica con un «florecimiento», que se entiende como un compromiso activo y saludable con el mundo al llevar una vida conforme a la virtud.

Cada teoría busca explicar qué es lo moralmente correcto con base, en el caso de la ética de la virtud, del carácter de los agentes que actúan; en el de la postura deontológica, en que las razones para actuar del agente se realicen conforme al deber que establece el Imperativo Categórico $\mathrm{y}$, en el caso del utilitarismo, en las mejores consecuencias, en términos del mayor placer para el mayor número de personas.

Las teorías normativas comparten características importantes: buscan ser universales, ofrecer un principio o una serie de principios que puedan aplicarse ante cualquier 
circunstancia y por cualquier agente que busque actuar de la forma correcta. Además, buscan ser objetivos en el sentido de que los principios que formulan son independientes de los agentes que los apliquen y su justificación no depende de elementos subjetivos. De esto se desprende que exigen cierta neutralidad del agente ${ }^{3}$.

Uno de los grandes retos a los que se enfrenta cualquier teoría normativa es que debe evitar la parcialidad de los agentes; también debería ser capaz de mostrarle a los agentes cuál es el curso de acción que tendría que tomar y, como consecuencia de esto, dar un cierto nivel de predictibilidad a las acciones ${ }^{4}$. Sin embargo, al tratarse de

${ }^{3} \mathrm{Al}$ menos en la formulación clásica presentada, pues la deontología que se basa en el agente sí toma en cuenta las circunstancias específicas de cada agente y establece sus deberes conforme a ello. No se profundiza en esta postura porque es una reformulación que responde a las objeciones hechas a la teoría original y lo que nos interesa en esta investigación es analizar los componentes de las teorías normativas y a qué responden, incluidas cuáles eran las metas de quienes las formularon por ocasión primera.

4 Puede tratarse de un nivel muy bajo de predictibilidad. Cristina Bicchieri señala, en Do the Right Thing, But Only if Others do So, la diferencia entre las expectativas normativas y las expectativas empíricas; entendidas las primeras como las expectativas que creemos que los otros tienen sobre lo que deberíamos hacer frente a una situación y las expectativas empíricas como lo que creemos que otros harían en nuestro lugar. Generalmente se piensa que ambas clases de expectativas tendrían que dirigirse hacia una misma acción, pero, tal como muestra el estudio de Bicchieri, en casos de corrupción, por 
sistemas generales, deben abstraer a los agentes de las circunstancias específicas en las que se encuentran; esta ha sido una crítica constante de pensadores como Bernard Williams (1987) o Jonathan Dancy (2004), quienes creen que una propuesta normativa está destinada a no dar cuenta de lo que se propone, pues deja de lado tanto las circunstancias del agente como las circunstancias de la situación a la que se enfrenta.

Parece que para que los requerimientos de universalidad y objetividad se cumplan, toda teoría normativa fallará al incorporar los elementos subjetivos relevantes para cada agente $y$ las circunstancias $o$ situaciones que rodean sus acciones. Las teorías normativas deben, además, suponer que hay algún proceso evaluativo previo a la acción que permite al agente decidir qué curso de acción es el más adecuado y es evidente que en muchas ocasiones - de hecho, la mayoría- no hay tiempo de realizar dicha reflexión o la reflexión puede verse afectada por factores externos. Además, existen casos extremos que suelen presentarse como contraejemplos para los principios que se desprenden de ellas.

Si Korsgaard está en lo correcto y la pregunta normativa se formula siempre desde la primera persona, si entra en juego en el momento en que un agente tiene que

ejemplo, las expectativas empíricas tienen más peso que las normativas para el agente que toma una decisión. 
decidir su curso de acción, entonces el terreno de lo moral está limitado a esas ocasiones y las teorías normativas no explican qué sucede en los casos de respuesta automática ${ }^{5} \mathrm{o}$ en las que el proceso de reflexión se ve afectado.

Cada teoría normativa da pautas para evaluar una acción como preferible ante otras acciones, en tanto que establece una definición de lo correcto y lo incorrecto. Nos permite evaluar, desde una perspectiva en tercera persona, nuestras propias acciones y las de los otros, por lo que debe incluir las respuestas a esperar tanto si cumplimos con las normas morales como si no lo hacemos. Las teorías normativas parecen perderse en el camino y pasar por alto propiedades básicas de los seres humanos en su búsqueda de principios universales.

Si el reduccionismo proviene de la búsqueda de principios universales y es evidente que siempre se cae en un reduccionismo en ética o que se establecen principios que caen con facilidad ante unos cuantos contraejemplos, entonces ¿qué caso tiene buscar establecer otro sistema normativo?

2. Apología de la Normatividad

${ }^{5}$ Aquí, un partidario de la ética de la virtud podría explicar respuestas automáticas como producto del hábito, que una vez perfeccionado, no necesita de un proceso deliberativo previo a la acción. 
Decíamos al inicio que la ética es un terreno escabroso y sus aproximaciones se enfrentan a múltiples problemas, de entrada, no es claro cómo distinguir los principios o normas morales de otro tipo de principios, como los prudenciales o los meramente convencionales. La idea de que la moral es importante en nuestras vidas es compartida por la mayoría, pero no se tiene una definición última acerca de qué es la moral, a qué refiere y cuáles son sus propiedades. Un punto de partida puede ser las intuiciones que compartimos sobre ella.

Las intuiciones pueden ser vistas como un recurso no lo suficientemente robusto para comenzar un estudio sobre la moral, sin embargo, es necesario establecer un punto de partida y el que se propone aquí consiste precisamente en las intuiciones que se comparten acerca de la moral y se concentra en la función que se le atribuye. Una perspectiva funcionalista acerca de la moral unida a un análisis exhaustivo de nuestras intuiciones morales nos da aspectos objetivos de los cuáles partir y asegura que nuestras intuiciones más profundas no son contradichas. Un sistema normativo moral que va en contra de nuestras intuiciones más básicas ${ }^{6}$ se enfrentaría a fuertes problemas en su

${ }^{6}$ La Teoría del Error, postulada por Mackie (1977) y desarrollada por Joyce (2006) señala que las características que le asignamos a la moral no coinciden con la realidad, que no existen Hechos morales que le den objetividad, importancia e inescapabilidad a la moralidad, pero explican 
práctica, y recordemos que la normatividad busca aplicar como guía para la acción.

Los estudios desde psicología y antropología nos permiten acceder a las intuiciones morales compartidas en distintos lugares del mundo ${ }^{7}$. Aquí haremos referencia a dos investigaciones, la rescatada por Víctor Kumar para analizar el Juicio Moral (2015) y la de Jonathan Haidt acerca de los objetos a los que se dirigen las normas morales (2012). En un primer momento podemos decir que la acción moral se concibe como aquella que va en contra de los meros intereses individuales, que se dirige a procurar bien de los otros y que, en este sentido, su función principal es regular el contacto social.

Desde la antropología y la sociología el análisis de las normas y su clasificación se concentra en las consecuencias que se tiene al romperlas. Cada sociedad o comunidad tiene

por qué les asignamos esas propiedades, en tanto la moral tiene una función evolutiva fundamental y tiene que aparecérsenos de esa forma para cumplirla. En un sentido dice que nuestras intuiciones acerca de la naturaleza de la moral son falsas, pero no se propone erradicarlas o cambiarlas. Aquí nos referimos a la experiencia que tenemos acerca de la moral y no a su naturaleza ontológica.

7 No nos enfocaremos en los problemas que plantea el Relativismo Moral, sin embargo, partimos de la idea de que las normas morales son lo suficientemente similares en todas partes como para encontrar patrones que nos den pistas importantes acerca de la moral. Lo que hace que las normas sean tan distintas tiene qué ver con las condiciones distintas que enfrenta cada sociedad. 
un código de conductas que puede estar establecido como alguna ley o sólo ser del conocimiento de todos los que cumplen ciertas características, como contar con una edad específica. Desde hace algunos años se han realizado múltiples investigaciones empíricas que buscan arrojar luz a la distinción entre convenciones sociales y normas morales. Las justificaciones que suelen dar los participantes de los estudios para distinguir las normas morales es que su violación genera daño, injusticia o va en contra de los derechos de los otros. Lo que parece distinguir las normas morales de otro tipo de normas es que su violación tiene reacciones muy serias, en tanto se les adjudica gran importancia. Las investigaciones muestran que lo que distingue a las normas morales es que son concebidas como serias, generales, independientes de la autoridad y objetivas $^{8}$ (Kumar, 2015).

La objetividad implica que no importa si se está o no de acuerdo con que deben seguirse las normas morales, no tienen qué ver con los estados subjetivos de cada persona o de un conjunto de personas para que sean inescapables. También implica que frente a desacuerdos morales las personas suelen pensar que una parte está en lo correcto y otra está equivocada. El criterio de generalidad se refiere a

${ }^{8}$ Kumar es muy específico en señalar que se trata de una propuesta conceptual y no ontológica, es acerca de cómo concebimos la moralidad, no de su naturaleza última. 
que son aplicables independientemente de las circunstancias y su seriedad refleja la importancia que tienen: las normas morales no deben ser tomadas a la ligera. El que sean independientes de la autoridad expresa cómo no se tratan de convenciones que puedan ser reguladas por alguna institución o algún personaje con poder: si las reglas de esa institución o personaje se modificaran, las normas morales se mantendrían sin cambios.

Según los estudios de Haidt, los Juicios Morales pueden clasificarse en 5 grupos principales conforme a lo que refieren (2012), están los más básicos, que involucran los criterios de daño o cuidado, generar daño a otro es considerado moralmente incorrecto en todas las poblaciones del mundo. También existe una noción compartida acerca de la justicia, los moralmente correcto se dirige a la justicia y la reciprocidad; un tercer grupo de juicios morales se dirige a la pertenencia al grupo y a la lealtad; el cuarto hace referencia a la autoridad y al respeto y, por último, tenemos la clasificación que versa sobre la puridad y la santidad ${ }^{9}$.

Aquí vale la pena señalar que los resultados presentados por Kumar y Haidt son descriptivos y no normativos, no pretendemos que lo sean. Sin embargo, nos permiten comprender un poco mejor cómo es que

${ }_{9}$ Haidt explora la posibilidad de agregar una sexta clasificación, que se dirige a la libertad y la opresión. Aquello que implique o genere opresión, que vaya en contra de la libertad, es considerado como moralmente incorrecto. 
funcionamos moralmente como sociedad, qué características le adjudicamos y hacia qué se dirigen nuestros juicios morales y profundizar en ellos. Desde allí es posible comenzar un análisis que parta de lo que es para trazar un deber que no vaya en contra de nuestras intuiciones fundamentales. Kumar expresa que su propuesta de las morales como serias, independientes de la autoridad, generales y objetivas es «una hipótesis en proceso, empíricamente revisable y potencialmente incompleta» (Kumar, 2015, 2896) y, a pesar de ello, es posible tomarla como un punto del cuál partir.

La características de la ética normativa, sus aspiraciones, coinciden con la forma en que tradicionalmente concebimos la moral y eso tendría que ser un indicador importante que nos lleve a seguir analizando la ética normativa, específicamente, a postular un sistema que pueda enfrentarse a las principales críticas y que, a pesar de todos los inconvenientes que se pueden desprender de su ser general y objetivo, nos proporcione guías para la acción y para la justificación de dichas acciones en -casi- todos los casos en los que nos enfrentemos a dilemas morales.

Por supuesto, será necesario establecer primero qué características de nuestro ser seres humanos resultan relevantes para actuar moralmente y qué vamos a tomar como aquello a lo que tendría que dirigirse la ética. Para el primer elemento necesitamos de la psicología moral, rama 
de la metaética, que trabaja en proyectos importantes acerca de, por ejemplo, cómo intervienen los elementos cognitivos y los afectivos en nuestros juicios morales. Para el segundo podemos explorar los estudios relativos al bienestar que también se llevan a cabo en la actualidad, en conjunto con la psicología, las ciencias cognitivas y la antropología, partiendo de la presuposición generalizada de que el contenido de la moral está al menos vagamente interesado en el bienestar humano (Svavarsdóttir, 1999).

Un sistema normativo como el que proponemos debe también aportar pautas básicas que puedan servir en los casos de la ética aplicada e, incluso, criterios que nos permitan comprender cuál es el papel específico que cumple la ética en esos casos. Seguir apuntando a establecer un sistema normativo en conjunto con las investigaciones en metaética es un proyecto que comienza y que, de ser exitoso, podrá darnos las bases necesarias para comprender un poco más de qué trata la moral y tener herramientas para defender en qué elementos tendríamos qué concentrarnos.

Aquí se apuesta por una teoría naturalista, funcionalista y que emplea tanto herramientas filosóficas fundamentales como investigaciones empíricas, pero propuestas normativas que vengan de otros lugares nos permitirán también sopesarlas, analizarlas y no dejar de lado el aspecto propiamente normativo de la moral, su dirección a la acción y, en general, su aplicación a nuestras 
vidas, también nos brindará herramientas que permitan un terreno común del qué partir en las discusiones de ética aplicada.

\section{El caso de la ablación femenina en África y lo que debemos aprender de él}

La ablación femenina se practica en un gran porcentaje de países africanos $\mathrm{y}$ ha generado conmoción en todo Occidente, así como importantes movimientos y estudios. Se estima que la ablación femenina continúa practicándose en 28 países de África (Young Eun Nam, 2018). La ablación femenina se practica de formas distintas, que van desde la extracción del prepucio del clítoris hasta la extracción total del clítoris y los labios menores. La edad en que se practica varía también, abarcando desde los primeros días de nacimiento hasta la pubertad. Aunque se practica en distintos lugares del mundo, poblaciones africanas tienen el mayor porcentaje de niñas y mujeres sometidas a tal procedimiento, situación que ha llamado la atención del mundo y generado la reprobación general de la práctica y de sus consecuencias.

Entre las principales consecuencias físicas, que varían en tanto varía el procedimiento, se encuentran serias infecciones, daños al sistema urinario, alteración de los ciclos menstruales, dispareunia y problemas obstétricos en tanto la cicatrización complica el parto; puede también 
generar infertilidad y sus consecuencias pueden llevar hasta la muerte. Además del daño físico, se reporta daño psicológico, la ablación resulta en irritabilidad, ansiedad, depresión y hasta alteraciones de personalidad.

Kouba y Muasher $(1985: 103,104)$ hacen un recuento de las razones que se dan para justificar la ablación, la mayor parte consecuencias de mitos tribales y de conocimiento erróneo respecto a cuestiones de salud y fisiología básica. La ablación femenina es especialmente practicada en comunidades musulmanas que, aunque no originaron la práctica, la adoptaron en tanto va en la línea de la virginidad y la castidad.

Los casos de ablación femenina suelen abordarse desde tres enfoques, el patriarcal, que denuncia la práctica como violencia fruto del patriarcado y que busca someter el cuerpo de las mujeres; el que parte de la tradición que busca comprenderla como un elemento fundamental de la cultura de la que es propia y desde allí busca herramientas que permitan modificar las creencias que la fundamentan y el tercer enfoque, que es el que nos ocupa aquí, es el que parte de una perspectiva universalista. Se enfoca en el daño que produce y la considera como una violación a los derechos humanos. Su justificación se basa en principios morales y apunta a la objetividad a la que aspira la normatividad en moral.

Los tres enfoques comparten una visión de África como un lugar que debe ser intervenido desde fuera, 
«salvado» de su propia barbarie. Lo que cambia son los métodos y las banderas que se emplean para el rescate: en el primer caso, las mujeres occidentales son las encargadas de liberar del sistema patriarcal a las mujeres africanas; en el segundo, los activistas occidentales son los encargados de «arreglar» las creencias erróneas de los pueblos africanos y en el tercero, una vez más son los ciudadanos de occidente quienes deben, en nombre de los derechos universales, erradicar la práctica.

Para erradicar la práctica se han llevado a cabo múltiples intervenciones en forma de seminarios informativos, también se ha legislado en su contra, imponiendo serias multas a quienes se involucren en el procedimiento y hasta encarcelamiento. Se ha buscado también dar apoyo económico, junto a información, a quienes son los encargados de llevar a cabo la ablación, generalmente mujeres mayores del grupo, con el fin de asegurar que dejen de participar en ella. Actualmente existen múltiples fundaciones dedicadas únicamente a combatir la ablación en África, como la Women For Africa Foundation, que busca que la práctica sea prohibida y castigada y que se generen programas de concientización en las comunidades por todos los medios posibles.

Las tres formas de abordar el problema parecen concebir a los africanos como inertes, como simples receptores de las acciones que vienen de fuera $y$ especialmente la tercera deja de lado aspectos 
fundamentales para comprenderla, sin incluir las voces de las mujeres africanas. En parte, se cree que las mujeres africanas no tendrían que tener un lugar importante en la discusión porque están impregnadas de falsas creencias heredadas por su cultura que pueden llevarlas a defender una práctica que, a ojos de Occidente, es completamente indefendible. La ablación femenina es un tema fundamental en las discusiones de bioética, de derechos humanos y de feminismo, pero poquísimas veces se escuchan voces de africanos ellas.

Resulta especialmente importante tener en cuenta que la ablación femenina responde a causas muy distintas en cada región y el acercamiento occidental suele dar por sentado que las causas pueden unirse todas y aplicarse a todas las regiones o que no existen excepciones que puedan generar algo distinto a juicios de reprobación total e inmediata para la ablación femenina.

Wairimũ Ngarũiya (Oyěwùmí, 2011: 179-197) presenta una historia diferente, en el grupo Gîkũy ũ en Kenia la práctica, con sus elementos propios de la comunidad, sirvió como un medio para formar parte de la comunidad, ser escuchadas y respetadas e incluso para ser reconocidas como parte del movimiento independentista que tuvo lugar en los años 40’s y 50's.

Ngarũiya describe cómo la práctica específicamente de las mujeres, denominada irua ria atumia, fue buscada por las mujeres de la región para ser reconocidas como miembros 
importantes de la sociedad. Los hombres pasaban por un proceso similar que simbolizaba su entrada al mundo adulto y les confería un lugar importante dentro del grupo, por lo que se celebró que la práctica se extendiera a las mujeres, quienes lo concebían como un privilegio.

Ambos procedimientos, femenino y masculino, incluían una serie de actividades de reclusión, formación e iniciación a las prácticas adultas del grupo: en el tiempo de curación, que duraba varios meses, se daba al grupo de iniciados información acerca del funcionamiento del grupo y se practicaban rituales que generaban una fuerte cohesión entre ellos. La irua ria atumia formaba parte de un grupo de prácticas que incluían la posibilidad de intercambiar parejas sexuales dentro del grupo que experimentó en conjunto el ritual.

Para los colonizadores, estas prácticas en conjunto fueron vistas desde el principio como primitivas e inmorales ${ }^{10}$, Ngarũiya sostiene que había intereses económicos y religiosos por parte de los colonizadores para oponerse al irua ria atumia, se pensaba que la práctica dificultaba la adhesión de las mujeres a la Iglesia y, al mismo tiempo, el que la duración del ritual completo tomara meses enteros intervenía con el flujo laboral, lo que llevó a su prohibición.

10 Resulta interesante que el equivalente masculino, el irua ria anake, la circuncisión masculina, no fue vista como contraria a la religión ni como inmoral. 
Pero continuó realizándose. Tomando incluso más peso que antes, era el primer paso, necesario, para poder involucrarse en las actividades anticoloniales. La irua ria atumia y la irua ria anake preparaban física $\mathrm{y}$ psicológicamente, fortaleciendo y dando un sentido de pertenencia, así como permitiéndole, a quienes pasaban por ellas, tomar juramentos fundamentales para el movimiento independentista. En caso de ser capturados, la experiencia del ritual les daba fortaleza ante las torturas de los colonizadores, que eran en muchos niveles aún más brutales que la ablación en sí, incluyendo, por ejemplo, sodomía con serpientes.

El trabajo de Ngarũiya presenta el ritual de forma integral, sin caer en generalizaciones y encuentra que, al menos en el caso de Kenia, la práctica puede significar lo contrario a lo que se pensaría desde una visión parcial. No la postula como algo deseable o algo que tenga que erradicarse, se enfoca en el papel de integración que significó para su comunidad y su potencialidad al momento de independizarse de Inglaterra.

Su objetivo principal es hacer notar que es necesario partir de la complejidad de las prácticas y asegurar que las voces locales son tomadas en cuenta; nos muestra una historia en la que la ablación femenina cumple un papel distinto y alcanza a mostrar que el enfoque limitado a la ablación femenina deja de lado elementos importantes como la colonización en sí, pues en el caso descrito se 
concebía a la ablación como terrible y bárbara, mientras se guardaba silencio respecto a las prácticas de tortura a la que los independentistas eran sometidos.

La forma de abordar la ablación femenina parece ignorar por completo sus circunstancias, así como pasar por alto las circunstancias particulares de cada región en la que se practica. Seguramente es el caso de que sirva como un mecanismo de control patriarcal en muchas de las zonas, pero enfocarse únicamente en un control patriarcal y no considerar el control colonial y sus consecuencias es caer en una doble moral.

Necesitamos investigaciones integrales que nos permitan un mayor conocimiento de la práctica y de sus circunstancias, similares a la llevada a cabo por Young Eun Nam (2018) que señala que en casos de Kenya, Uganda y Etiopía, las mujeres que no son circuncidadas son aún consideradas «impuras» e «inmaduras», que existen importantes intereses económicos por parte de quienes son los encargados de llevar a cabo la práctica y que existe una correlación entre la falta de recursos económicos y la inescapabilidad del ritual para muchas mujeres en éstas regiones.

Es urgente comenzar a construir críticas que sean autónomas y que estén localizadas geográfica, histórica y culturalmente y que partan de lugares alternos a los hegemónicos, en cualquiera de su presentación. La oposición a la ablación femenina resulta ser un lugar común 
para muchos y para poder abordarla adecuadamente será necesario analizarla de forma integral $y$, sobre todo, asegurar que testimonios como el de Ngarũiya son tomados en cuenta.

El caso de la ablación femenina nos enseña algo fundamental que el proyecto de generar un sistema normativo debe tomar en cuenta, esto es, que debemos cuidar en enfoque al que se aplica y que no podemos, al tomar casos específicos, y especialmente casos tan polémicos, ignorar sus circunstancias estructurales y materiales.

En un primer principio las intervenciones que apelan a los derechos humanos, en tanto se presentan como universales, objetivos e inescapables, podrían pasar como parte de una normatividad moral, pero no pueden aplicarse sin más. Un sistema normativo puede establecer que aquello que genera daño e injusticia es incorrecto, pero no nos da la información acerca de cómo debe aplicarse. El mismo sistema, visto en una perspectiva más completa, señalaría que las acciones colonialistas en África son incorrectas en muchos sentidos, con los mismos criterios dados por la teoría normativa.

\section{Conclusiones}

El texto presenta las razones a favor de que se retomen lo estudios en ética normativa y que se apueste por formular 
sistemas normativos que puedan enfrentarse a las principales críticas que se les hacen tradicionalmente. Esto en tanto permite conservar el carácter práctico de la ética y dar una base teórica robusta a las discusiones específicas de ética aplicada $\mathrm{y}$ en tanto el proyecto normativo se corresponde con las intuiciones más fundamentales que compartimos acerca de la moral.

Defiende además que la investigación normativa tendría que retomarse y vincularse con las discusiones en metaética y en ética aplicada. Dicha vinculación nos permitirá comprender nuestra moralidad y establecer a qué se dirige, así como sus alcances y límites. A su vez, la ética normativa daría un sustento teórico robusto a las discusiones en ética aplicada, conservando el carácter normativo que no tiene la metaética, por concentrarse en los debates teóricos, pero nutriéndose a la vez tanto de su aplicación a casos concretos y de los descubrimientos descriptivos de la moralidad.

Por último, traza una advertencia para la creación y específicamente la aplicación de los sistemas normativos que se generen, pues acciones como las de organizaciones que buscan erradicar la práctica de la ablación femenina en comunidades africanas usan como bandera nociones que se asemejan a las que, en teoría, tendrían las teorías normativas, en tanto aspiran a ser generales, objetivas, serias e independientes de la autoridad. Una teoría normativa no puede aplicarse sin tener a la par un 
conocimiento de las circunstancias del caso, eso no implica que la teoría normativa sea incorrecta o insuficiente, pues lo que proporciona son bases generales que apuntan al bienestar de las personas.

Una propuesta normativa no puede contener toda la información, pero puede, aplicada junto a un conocimiento de las circunstancias, mostrar qué acciones irían conforme a lo correcto moralmente hablando y justificarían las acciones que se dirijan a ello.

\section{Bibliografía}

Bicchieri, C. \& Xiao, E. (2009), Do the right thing: but only if others do so. Journal of Behavavioral Decision Making 22, 191-208.

Dancy, J. (2004). Ethics without principles. Oxford: Clarendon Press.

Dreier, J. (2006). Contemporary Debates in Moral Theory, U.K.: Blackwell Publishing.

Haidt, J. (2012). The Righteous Mind, Why good people are divided by politics and religion, USA: Pantheon Books.

Joyce, R. (2006). The evolution of Morality. Massachusetts: MIT Press. 
Korsgaard, C. (2000). Las fuentes de la normatividad, Laura Lecuona \& Laura Manríquez (trad.). México: IIF/UNAM.

Kouba, L. J. \& Muasher, J. (1985). Female Circumcision in Africa: An Overview. African Studies Review 1 (28), 95110.

Kumar, V. (2015). Moral judgment as a natural kind. Philosophical Studies 11 (172), 2887-2910.

Mackie, J.L. (1977). Ethics: Inventing wright and wrong. USA: Penguin Books.

Miller, A. (2003). An Introduction to Contemporary Metaethics. UK: Polity Press.

Mohanty, Ch. et. al. (eds.). (1991). Third World Women and the politics of feminism. USA: Indiana University Press.

Shaer, G. (ed.). (2012). Ethics: Essential Readings in Moral Theory. U.K.: Routledge.

Svavarsdóttir, S. (1999). Moral Cognitivism and Moral Motivation. The Philosophical Review 2 (108), 161-219.

Oyěwùmí, O. (ed.). (2011). Gender Epistemologies in Africa Gendering Traditions, Spaces, Social Institutions, and Identities, USA: Palgrave Macmillan.

Williams, B. (1987). Ethics and the Limits of Philosophy. Ethics 4 (97), 821-833.

Young Eun Nam. (2018). The power structure in the perpetuation of female genital cutting in Kenia. Asian Journal of Women's Studies 1 (24), 128-139. 